\title{
Diffusons, Locons, Propagons: Character of Atomic Vibrations in Amorphous Si
}

\author{
Philip B. Allen \\ Department of Physics and Astronomy, State University of New York, Stony Brook, New York 11794-3800 \\ Joseph L. Feldman \\ Naval Research Laboratory, Washington DC 20375-5345 \\ Jaroslav Fabian \\ Department of Physics, University of Maryland at College Park, College Park, Maryland 20742-4111 \\ Frederick Wooten \\ Department of Applied Science, University of California at Davis, Davis, California 95616
}

Numerical studies of amorphous silicon show that the lowest $4 \%$ of vibrational modes are plane-wave like ("propagons") and the highest $3 \%$ of modes are localized ("locons"). The rest are neither plane-wave like nor localized. We call them "diffusons." Since diffusons are by far the most numerous, we try to characterize them by calculating such properties as wavevector and polarization (which seem not to be useful), "phase quotient" (a measure of the change of vibrational phase on between first neighbor atoms), spatial polarization memory, and diffusivity. Localized states are characterized by finding decay lengths, inverse participation ratios, and coordination numbers of the atoms participating.

61.43.Dq, 63.50.+x, 66.70.+f

\section{INTRODUCTION}

Vibrational properties of disordered media have been reviewed by various authors, in particular Elliott and Leath (1975), Weaire and Taylor (1980), Visscher and Gubernaitis (1980), and Pohl (1998). Here we review and present new results in a program of numerical study of vibrations of amorphous Si. Among the new results not contained in earlier reviews are theoretical treatments of heat conductivity and thermalization rates in glasses.

Harmonic normal modes of vibration can be rigorously classified as extended (E) or localized (L). In three dimensions the vibrational spectrum has sharp $\mathrm{E} / \mathrm{L}$ boundaries ("mobility edges") separating these two kinds of modes.

There is another boundary, not sharp, which Mott called the "Ioffe-Regel limit" and which we call the "IoffeRegel crossover." This P/D boundary separates the spectrum into a region with ballistic propagation $(\mathrm{P})$ where wavevector is a reasonably good quantum number and a region with only diffusion (D) where wavevector cannot be defined but states are still extended. In region $\mathrm{P}$, wave-packets can travel at sound velocity over distances of at least two or three interatomic spacings before scattering from disorder (Allen and Kellner, 1998). The distance of ballistic propagation is the mean free-path $\ell$.
In region $\mathrm{D}$, only diffusive propagation occurs over any meaningful distance, and the concepts of mean free path and wavevector lose usefulness. Although it may seem natural that the Ioffe-Regel crossover should be close to the mobility edge (Alexander, 1989), it is not true for the models we have studied. Indeed, Mott and Davis (1971) emphasize the non-coincidence. Both regions $\mathrm{P}$ and D lie in the extended (E) part of the spectrum.

The non-coincidence of the $\mathrm{E} / \mathrm{L}$ boundary and the $\mathrm{P} / \mathrm{D}$ boundary means that the spectrum has three kinds of states. We find useful the terminology "propagon, diffuson, and locon" given in Fig. 2. The term "phonon" is avoided because of disagreement about what it means in a glass.

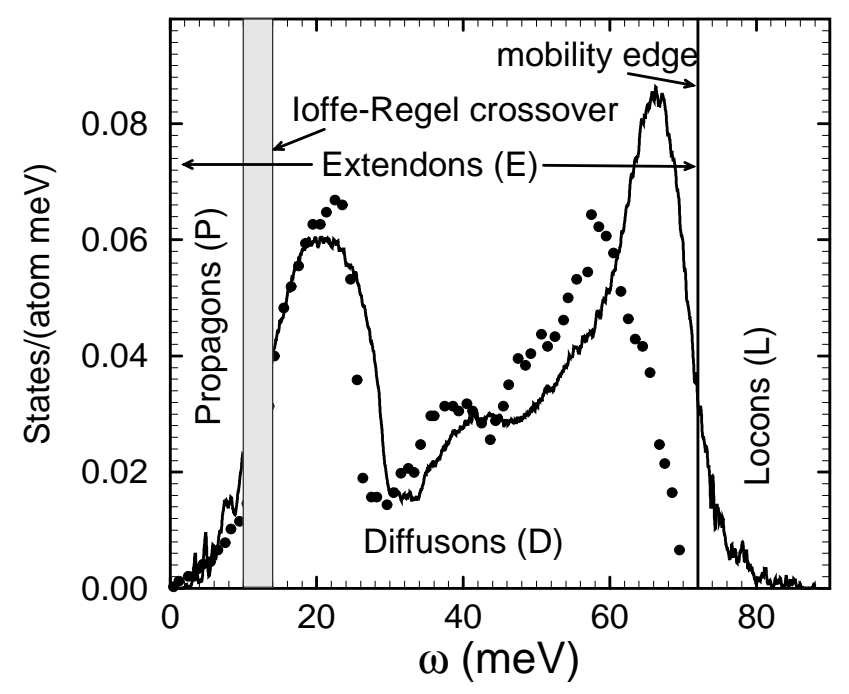

FIG. 1. Density of vibrational states from the 4096-atom model compared with data from Kamitakahara et al. (1987).

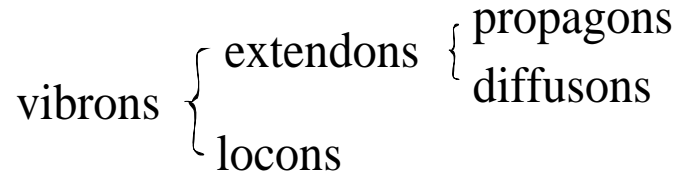


FIG. 2. Taxonomy of vibrations in glasses

We instinctively seek additional labels, to replace the detailed classification scheme by wavevector and branch so useful in crystals. Here we attempt to characterize as completely as possible the dominant diffuson portion of the spectrum, but fail to find useful sub-categories. As already noticed by Kamitakahara et al. (1987), all diffuson modes of a given frequency have essentially indistinguishable displacement patterns.

We study amorphous silicon in harmonic approximation, using a model we believe to be very realistic. As shown in Fig. 1, the E/L boundary is near the top of the spectrum, with only $3 \%$ of the modes localized. The $\mathrm{P} / \mathrm{D}$ boundary is near the bottom of the spectrum, with only $4 \%$ of the modes ballistically propagating. Diffusons fill $93 \%$ of the spectrum. We do not think this is special either to our model or to amorphous Si. Other models for amorphous Si (Kamitakahara et al. 1987, Lee et al., 1991, Nakhmanson and Drabold, 1998) agree that the E/L boundary occurs near the top of the spectrum. Similar results are found for other glasses (Bouchard et al. 1988; Feldman and Kluge, 1995; Cobb, Drabold, and Cappelletti, 1996; Taraskin and Elliott, 1997; Carles et al., 1998), and model systems (Sheng and Zhou, 1991; Sheng, Zhou, and Zhang, 1994; Schirmacher, Diezemann, and Ganter, 1998), with localized states occurring only near the top of the spectrum or in tails near gaps in the vibrational densities of states. For 3 -d systems with artificially large disorder, the mobility edge can be moved down to the middle of the spectrum (Canisius and van Hemmen, 1985; Fabian and Allen, 1996). The position of the $\mathrm{P} / \mathrm{D}$ boundary near the bottom of the spectrum is widely accepted.

\section{LOW-FREQUENCY ANOMALIES}

Our study of normal modes by exact diagonalization on finite-size systems inherently lacks information at low frequency. For this reason, the present paper ignores the well-known but only partially understood low-frequency anomalies in vibrational properties of glasses. Our previous work (Feldman, Allen, and Bickham, 1999) argues that the homogeneous network models we use probably contain no low-frequency anomalies. For completeness, we give here a brief catalog and point to sources for further information.

"Two-level", or "tunneling" systems were introduced by Phillips (1972) and Anderson, Halperin, and Varma (1972) motivated by experimental discoveries by Zeller and Pohl (1971). The predictive strength of this concept is beyond question, but the physical objects the theory invokes remain elusive. A review was given by Phillips (1987).

The phrase "boson peak" refers to a low frequency feature seen by Raman scattering (Stolen, 1970; Jäckle,
1981) in many glasses, which is correlated with the occurrence of "excess modes" in specific heat and other spectroscopies. There are many candidate explanations for these modes. One unified view, introduced by Karpov, Klinger, and Ignat'ev (1983) is called the "soft potential model." This model holds that glasses generically have anharmonic regions, modelled as double-well potentials. These give rise to two-level systems , relaxational behavior, and quasi-localized or resonant harmonic normal modes. The last are a logical candidate for the excess modes. The subject was reviewed by Parshin (1994). Our models contain some quasi-localized modes at low frequencies, as is mentioned further in Sec. VIII.

"Floppy modes" (Phillips, 1980; Thorpe, 1983) and the daughter concept of "rigid unit modes" (RUMs: Dove et al. 1996; Trachenko et al. 1998) refer to low-frequency modes which have zero restoring force in nearest-neighbor central-force models. Constraint counting algorithms provide methods of estimating the numbers of such modes. They are expected to be quasilocalized in harmonic approximation, but intrinsically highly harmonic. Such modes probably do not play any important role in amorphous $\mathrm{Si}$ because of the overconstrained coordination.

Finally, experimental evidence shows that amorphous Si contains usually fewer two-level-type excitations than most glasses, and that samples with essentially zero such excitations can be prepared by treatment with hydrogen (Liu et al. 1997).

\section{THE MODEL}

Amorphous Si is an over-constrained network glass. By one usual definition, it is not a glass since it is not produced by a glass transition upon cooling. Instead, thin films are condensed on cold substrates. When the film gets too thick, crystallization cannot be prevented. The absence of a glass transition can be attributed to good kinetic properties of a single-component system with one strongly-preferred bonding arrangement. To our mind, this just means that amorphous $\mathrm{Si}$ is simpler than most glasses, which for our purposes is more of an advantage than a disadvantage. With obvious caution required, we think that most of the properties we shall discuss can be regarded as typical of most glasses. 


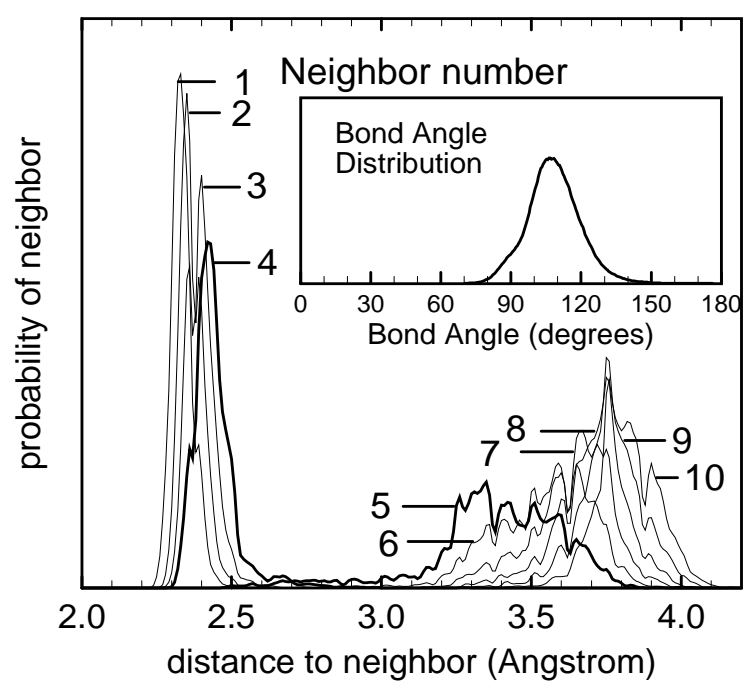

FIG. 3. Distribution of $n$th neighbors in the 4096-atom model of amorphous silicon. Fourth and fifth neighbors are shown in bolder lines. Note the small number of distant fourth or close fifth neighbors. The inset shows the distribution of bond angles averaged over all pairs of bonds with a common center and distances less than $2.6 \stackrel{\circ}{A}$.

For amorphous Si we use atomic coordinates generated using the algorithm of Wooten, Weiner, and Weaire (1985). We have studied models containing 216, 1000, and 4096 atoms, contained in cubic boxes of side 16.5, 28 , and $44 \AA$, respectively, and continued periodically to infinity. All models are built using the Keating potential (Keating, 1966) and then subsequently re-relaxed to a local minimum of the Stillinger-Weber potential (Stillinger and Weber, 1985). Different models differ in minor details, both for ordinary statistical reasons and because the algorithm was implemented slightly differently in each case. In this section we present structural properties of a 4096-atom model. This model has larger distortions from tetrahedral form than some of our other models, with 102 four-membered rings. Figure 3 shows neighbor distributions $\mathcal{P}_{n}(\omega)$ The distribution $\mathcal{P}_{1}$ for the first neighbor is calculated by measuring the distance to the closest neighbor of each atom; $\mathcal{P}_{2}$ is found from the distances to the second closest neighbor. Note that the first four neighbors distribute tightly within $\approx 0.1 \AA$ to the crystalline first neighbor distance, $2.35 \AA$. The next 12 neighbors in crystalline $\mathrm{Si}$ are at $3.84 \AA$, a number fixed by the bond length $(2.35 \AA)$ and the tetrahedral bond angle $\left(109.5^{\circ}\right)$. In our model of amorphous $\mathrm{Si}$, the closest of the next 12 (neighbor number 5 ) lies roughly between 3.2 and $3.7 \AA$, while the farthest of these 12 (neighbor number 16 , not shown) lies roughly between 3.9 and $4.4 \AA$. This reflects a flexibility in the bond angles, with values distributed between $90^{\circ}$ and $125^{\circ}$, as shown in the inset to Fig. 3. The third set of neighbors in crystalline $\mathrm{Si}$ is 12 atoms at $4.50 \AA$. This is determined by the fact that diamond structure has a dihedral angle of $60^{\circ}$, with all rings of the 6-member "chair" type. Rotation of the dihedral angle to $0^{\circ}$ ("boat" type rings) reduces the third neighbor distance to $3.92 \AA$. Our model shows no gap at all between second shell (neighbors 5-16) and third shell (neighbors 17-28), consistent with random dihedral angles.

The sum $\sum \mathcal{P}_{n}$ over all $n$ gives the radial distribution function $g(r)$, plotted in Fig. 1 and compared with the experiments of Kugler et al. The close agreement is one measure of the realism of the model. However, much of the structure of $g(r)$ seems only to reflect atom density and nearest neighbor distance, so it may not be a very stringent test.

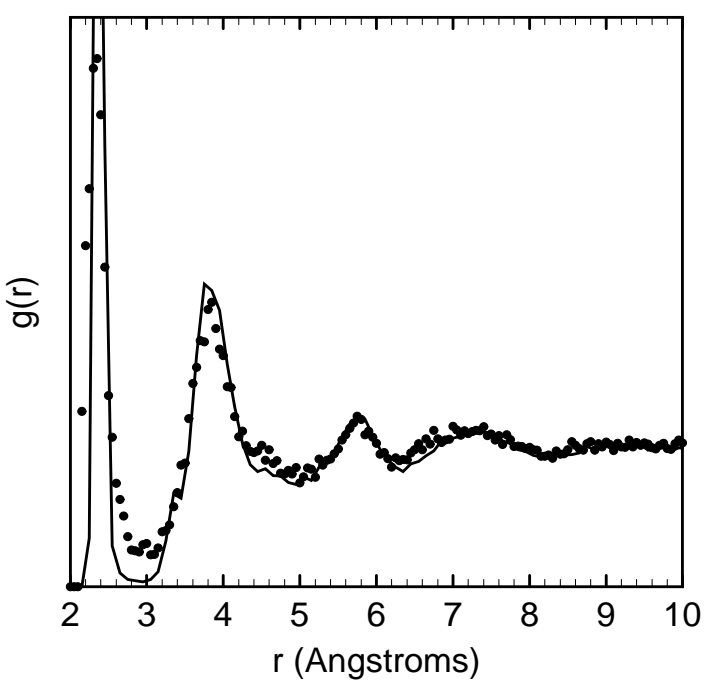

FIG. 4. Radial distribution function obtained by summing the $n$th neighbor distributions of Fig. 3 over all $n$, and dividing out a factor $r^{2}$. The data are from a neutron diffraction experiment by Kugler et al. (1993).

\section{VIBRATIONAL FREQUENCIES}

The other aspect of the model is the interatomic forces. We chose the model of Stillinger and Weber (1985) which is designed to work for the liquid as well as crystalline state. This required us to relax the coordinates from Wooten to a minimum of the Stillinger-Weber potential. The stability of the minimum is proven by the positivity of all eigenvalues $\omega^{2}$ of the dynamical matrix. The eigenfrequency distribution is shown in Fig. 1. Qualitatively satisfactory agreement is found with the neutron scattering data of Kamitakara et al. (1987). A similar overestimate of vibrational frequencies is made when Stillinger-Weber forces are applied to crystalline $\mathrm{Si}$, so we think the discrepancies should be attributed to the forces rather than the atomic coordinates. 


\section{LOCALIZED STATES}

The definition of a localized state is exponential decay of the eigenvector with distance from some center $\vec{R}_{0}$ :

$$
\left|\vec{\epsilon}_{i}\left(\vec{R}_{n}\right)\right| \propto \exp \left(-\left|\vec{R}_{n}-\vec{R}_{0}\right| / \xi_{i}\right)
$$

This defines the localization length $\xi_{i}$ of the $i$-th normal mode, if the decay is observed. Fig. 5 shows selected modes, showing the very different character of modes from the $\mathrm{D}$ and the $\mathrm{L}$ portions of the spectrum.

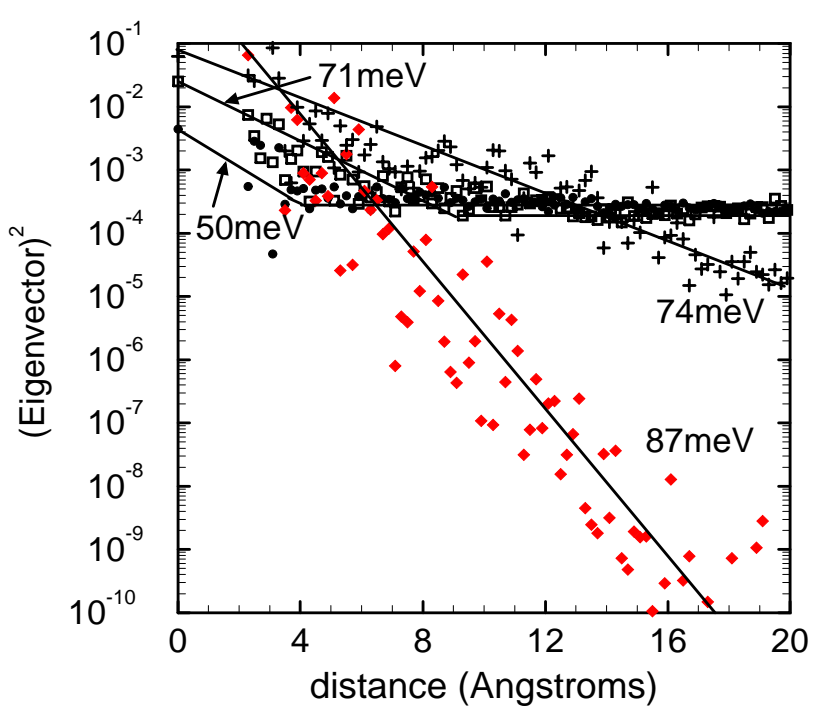

FIG. 5. Spatial decay of vibrational eigenvectors $\epsilon_{i}$. Solid lines are guides to the eye. For each mode $i$ the atom with the largest $|\epsilon|^{2}$ is located, and taken as the origin. The plot shows values of $\left|\epsilon\left(\vec{R}_{n}\right)\right|^{2}$ averaged over spherical shells of $\left|\vec{R}_{n}\right|$ of width $\delta R=0.2 \AA$. Results shown for $\omega=50 \mathrm{meV}, 71 \mathrm{meV}$, $74 \mathrm{meV}$, and $87 \mathrm{meV}$, are averages over 3, 4, 2, and 2 modes respectively. Modes at $50 \mathrm{meV}$ and $71 \mathrm{meV}$ have mean square eigenvector near $1 /(N=4096)$ throughout the cubic cell of length $44 \AA$, with enhanced values only in spatial regions of small measure. These modes are extended, as are all the modes below the mobility edge at $73 \mathrm{meV}$. Modes at $74 \mathrm{meV}$ and $87 \mathrm{meV}$ have mean values of $\left|\epsilon\left(\vec{R}_{n}\right)\right|^{2} \approx \exp \left(-2\left|\vec{R}_{n}\right| / \xi\right)$ with localization length $\xi=4.7 \AA$ and $1.5 \AA$ respectively.

There is still controversy concerning the location of the mobility edge in glasses. Unfortunately, most experiments shed little light, since measured spectral properties, being averages over a macroscopic region, do not differentiate between localized and delocalized states. Heat conductivity $\kappa(T)$ is the property most strongly affected by localization. We think the measured $\kappa(T)$ strongly supports our placement of the $\mathrm{P} / \mathrm{D}$ and $\mathrm{E} / \mathrm{L}$ boundaries near the lower and upper edges of the spectrum. This will be discussed in the next section.

If eigenvectors are calculated, then no special tricks are needed to locate the theoretical mobility edge in models of amorphous silicon. A model with 216 atoms was large enough to locate the E/L boundary between 71 and 73 $\mathrm{meV}$; the precise location varies somewhat from model to model. We have made some experiments with artificially enhanced disorder, randomly scaling half of the masses by a factor of 5 . This pushes the $\mathrm{E} / \mathrm{L}$ boundary into the middle of the spectrum where it becomes more blurred by size effects. Localized states of "pure" amorphous $\mathrm{Si}$ are easily recognized. They are trapped in especially defective regions. This was discovered (Fabian, 1997b) by defining a local coordination number $Z_{a}$, the number of neighbors of atom $a$ at $\vec{R}_{a}$. The following arbitrary definition of neighbor suffices:

$$
Z_{a}=\sum_{b} w\left(\left|\vec{R}_{a}-\vec{R}_{b}\right|\right)
$$

where $w(r)$ is 1 for $r<2.35 \AA$, 0 for $r>3.84 \AA$, and continuous and linear in between. This gives an average coordination of 4.7 neighbors. The "mode average coordination number" is defined as

$$
Z_{i}=\sum_{a} Z_{a}\left|\vec{\epsilon}_{i}(a)\right|^{2}
$$

Most modes have $Z_{i}$ near average, but localized modes mostly collect at regions with significantly higher coordination, as shown on Fig. 6.

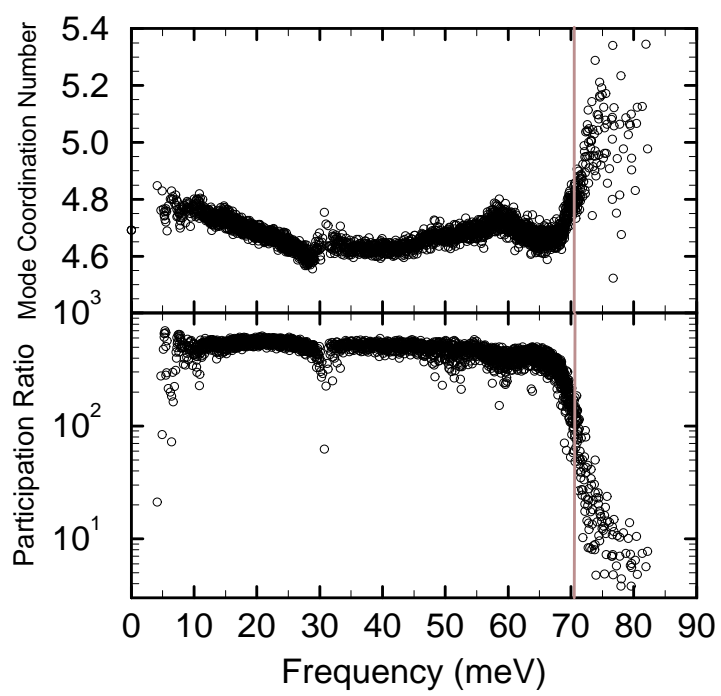

FIG. 6. The mode average coordination number (Eqn. 3), and the participation ratio (on a logarithmic scale), calculated for a model with 1000 atoms.

We have seen the mobility edge in many independent calculations.

1. The participation ratio $p_{i}$ (Bell and Dean, 1970), plotted on Fig. 6, gives the number of atoms on which the mode has significant amplitude. For the $\mathrm{E}=\mathrm{P}+\mathrm{D}$ part of the spectrum, the value hovers near 500 for a model with 1000 atoms. 
2. The diffusivity $D_{i}$ drops to zero at the mobility edge. This is shown in the next section.

3. Sensitivity to boundary conditions is also discussed in the next section.

4. Level spacing distributions have been computed for diffusons and locons (Fabian, 1997b). As expected, diffusons obey Wigner-Dyson statistics, while locons obey Poisson statistics.

5. Eigenvector self-correlation functions were carefully studied in a 4096-atom model by Feldman et al. (1998).

6. Many quantities $q_{i}$ which can be evaluated for each mode $i$ seem to depend only on $\omega_{i}$ for diffusons, but become mode-specific for locons. The ones we have looked at are:

(a.) The mode-average coordination number, plotted on Fig. 6.

(b.) The phase-quotient parameter, discussed in section VII.C.

(c.) Bond-stretching parameters (Fabian and Allen, 1997).

(d.) Grüneisen parameters for both volume and shear deformations (Fabian and Allen, 1997 and 1999).

\section{HEAT CONDUCTIVITY AND DIFFUSIVITY}

Theoretical interpretation of heat conduction of glasses has been contentious. Below the "plateau" region (typically $5-30 \mathrm{~K}$ ) it is agreed that heat is carried by ballistically propagating low-frequency modes (the $\mathrm{P}$ region of the spectrum). Above the plateau, $\kappa(T)$ rises, approaching at room temperature a constant value which is typically smaller than the crystalline value (a decreasing function of $T$ at room temperature.) A rigorous consequence (Allen and Feldman, 1993) of the Kubo formula (Kubo, 1957) and the harmonic approximation is the relation

$$
\kappa(T)=\frac{1}{V} \sum_{i} C\left(\hbar \omega_{i} / 2 k_{B} T\right) D_{i},
$$

where $\mathrm{C}(\mathrm{x})$ is the specific heat of a harmonic oscillator $(x / \sinh (x))^{2}$ and $D_{i}$ is the "diffusivity" of the $i$-th normal mode of frequency $\omega_{i}$, given by

$$
D_{i}=\frac{\pi V^{2}}{3 \hbar^{2} \omega_{i}^{2}} \sum_{j \neq i}\left|S_{i j}\right|^{2} \delta\left(\omega_{i}-\omega_{j}\right)
$$

where $S_{i j}=<i|S| j>$ is the intermode matrix element of the heat current operator. Eq. 6 also emerges, with $D_{Q}$ equal to $v_{Q}^{2} \tau_{Q} / 3$, from the Peierls-Boltzmann phonongas model (Gurevich, 1986) of transport in crystals. The latter model is only justified if the mean free-path $\ell_{Q}=$ $v_{Q} \tau_{Q}$ is longer than a wavelength.

It was noticed by Birch and Clark (1940), and by Kittel (1948) that in glasses $\kappa(T)$ at $T>20 \mathrm{~K}$ could be interpreted as the specific heat $C(T) / V$ multiplied by a temperature-independent diffusivity $\bar{D}$ of order $a^{2} \omega_{D} / 3$ where $a$ is an interatomic distance. In the phonon-gas model, this would correspond to $\ell \approx a$, too small to justify use of the model. The success of this observation implies that the dominant normal modes in a glass are of the $\mathrm{D}$ variety, not $\mathrm{P}$ because $\mathrm{P}$ implies $\ell \gg a$, and not $\mathrm{L}$ because $\mathrm{L}$ implies $D=0$ until anharmonic corrections are added which make $D$ depend on $T$. This successful (and we believe, essentially correct) interpretation lost favor after Anderson localization was understood, because a misconception arose that the $\mathrm{P} / \mathrm{D}$ boundary (which certainly lies low in the spectrum of a glass) should lie close to the $\mathrm{E} / \mathrm{L}$ boundary.

Our numerical calculations of $D_{i}$ are shown in Fig. 7. Also shown are values of $D_{i}$ from a formula of Edwards and Thouless (1972),

$$
D_{i}=L^{2} \Delta \omega_{i}
$$

where $\Delta \omega_{i}$ is the sensitivity of the eigenenergy to a twist of the boundary condition. We have simply used for $\Delta \omega_{i}$ the change in $\omega_{i}$ when boundary conditions are changed from periodic to antiperiodic. The actual definition is probably

$$
\Delta \omega_{i}=\lim _{\phi \rightarrow 0}\left[\frac{\pi^{2}}{\phi^{2}} \Delta \omega_{i}(\phi)\right]
$$

where $\Delta \omega_{i}(\phi)$ is the shift when the phase is twisted by $\phi$. Antiperiodic boundary conditions correspond to $\phi=\pi$, while $\phi=2 \pi$ returns to periodic boundary conditions with $\Delta \omega_{i}(2 \pi)=0$. Therefore our calculation, which is the only one easily accessible for us, gives a probable upper bound to $D_{i}$ for each mode $i$. Inspection of Fig. 7 shows that with this interpretation, the two calculations agree reasonably well. Both go to zero at the mobility edge, and both become large and ragged in the $\mathrm{P}$ region below $10 \mathrm{meV}$. The raggedness comes from the sparseness of the eigenstates at low $\omega$, and the large values reflect the onset of ballistic propagation. In the $\mathrm{D}$ region above $12 \mathrm{meV}$, values have collapsed to the range of $1 \mathrm{~mm}^{2} / \mathrm{s}$, which corresponds to $\omega_{D} a^{2} / 3$, with $\omega_{D}$ set to $50 \mathrm{meV}$ and $a=2 \AA$. This diffusivity is well below any value that could be allowed in a phonon-gas picture, and agrees with the measured $\kappa(T)$ (Allen and Feldman, 1990; Feldman et al., 1993). The peak of $D_{i}$ around $33 \mathrm{meV}$ corresponds to a feature in the "phase-quotient" that will be discussed in section VII.C. Similar results for vitreous $\mathrm{SiO}_{2}$ have been reported by Feldman and Kluge (1995). 


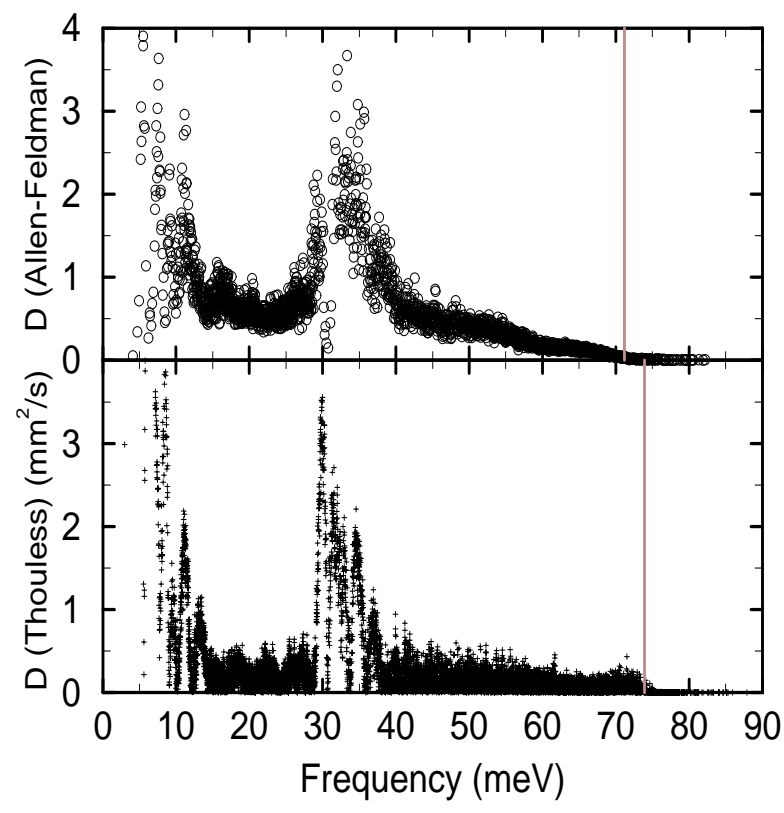

FIG. 7. Diffusivity versus frequency (Feldman et al., 1993, Fabian, 1997b) calculated for a 1000 atom model by Eq. 5 . and for a 4096 atom model by Eq. 6. Mobility edges, shown as vertical lines, are slightly different in these two models.

When $\kappa(T)$ is calculated from Eq. 4, using the values of $D_{i}$ from Fig. 17, the results, shown in Fig. B agree roughly with the data at higher temperatures. At low temperature it is necessary to have an additional source of heat current, the ballistically propagating long-wavelength modes. In Fig. 8, this has been added in a thoroughly ad hoc fashion. We have simply assumed a Debye spectrum for the modes with $\omega<\omega_{0}=3 \mathrm{meV}$, and a temperature independent diffusivity $D(\omega)=D_{0}\left(\omega_{0} / \omega\right)^{2}$. There is no theoretical justification for this. In principle, the temperature-independent diffusivity caused by glassy disorder should take the Rayleigh $\left(\omega_{0} / \omega\right)^{4}$ form at low $\omega$, and one needs a stronger type of scattering, inelastic, and therefore $T$-dependent, to match the data. However, the $\left(\omega_{0} / \omega\right)^{2}$ behavior has been seen at intermediate frequencies, both experimentally (Sette et al. 1998) and numerically (Dell'Anna et al. 1998; Feldman et al. 1999), so we have used this simpler fitting proceedure to match the data.

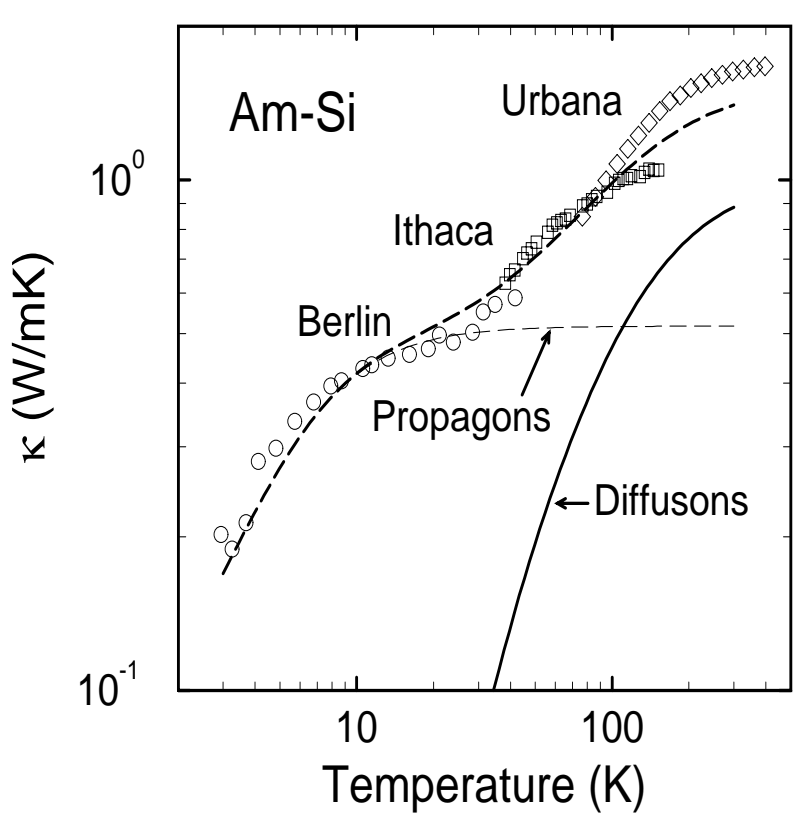

FIG. 8. Thermal conductivity $\kappa$ versus temperature $T$ measured for amorphous silicon (circles: Pompe and Hegenbarth, 1988; squares: Cahill et al., 1989; diamonds: Cahill et al., 1994). The bold line is a calculation from the diffusivity values shown in Fig. 7 for a 1000 atom model using Eq. 4. The thin dashed line is a calculation from modes at low energy assuming that their diffusivity obeys an $\omega^{-2}$ law. The bold dashed line is the sum of these two contributions.

Our most important conclusion is that the "reincrease" of thermal conductivity above the plateau region is attributable to heat carried by "diffuson" modes in much the way imagined by Birch, Clark, and Kittel, and that the plateau is a simple crossover region, not requiring any new physics to explain. In particular, we believe that "excess modes" (also known as a "Boson peak") is not a necessary ingredient to explain the plateau. Amorphous silicon seems to lack these "excess modes" but still to have a plateau.

\section{THERMAL EQUILIBRATION}

There is some evidence suggesting that vibrations in glasses, if disturbed from equilibrium, may return very slowly. For amorphous silicon, experiments were reported by Scholten and Dijkhuis (1996) and by Scholten, Akimov, and Dijkhuis (1996). Our investigations show that if the disturbance is not too large and is purely vibrational, then the rate of return to equilibrium should be as fast, if not faster, than in a corresponding crystal. Surprisingly, we find that this is true both in the locon and in the diffuson portion of the spectrum, contradicting a view (Orbach, 1996) supported by fracton theory (Alexander, 1989) that localized vibrations must equilibrate slowly. 


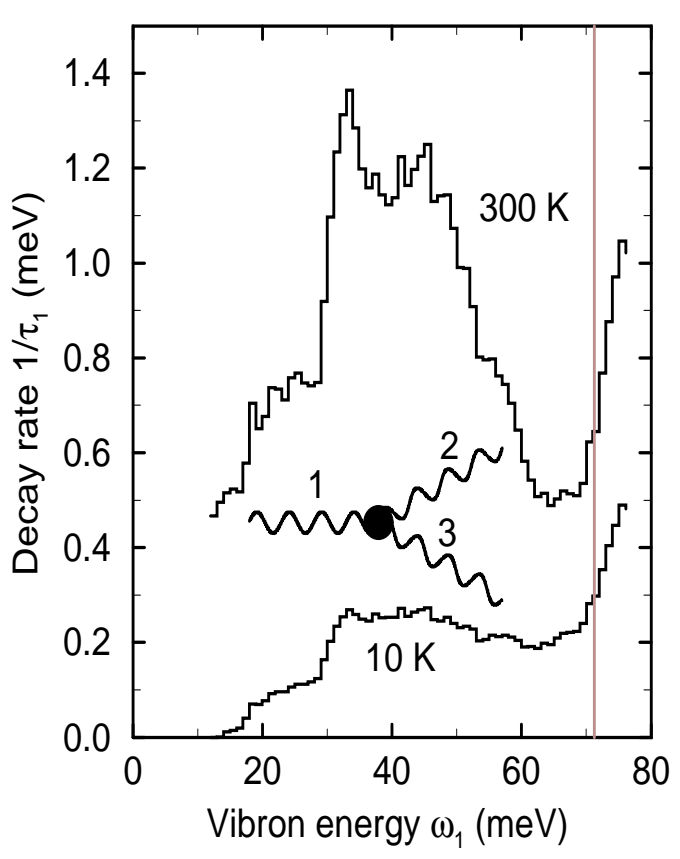

FIG. 9. Decay rates calculated by anharmonic perturbation theory for amorphous Si. The inset shows the process diagrammatically.

In thermal equilibrium, the harmonic vibrational eigenstates have average population given by the BoseEinstein distribution. Fabian and Allen (1996) used anharmonic perturbation theory to calculate the inverse lifetime or equilibration rate $1 / \tau$ by which a vibrational state returns to the Bose-Einstein distribution if driven out of equilibrium. Their results are shown in Fig. 9 . The validity of the perturbation theory is confirmed both by the smallness of the ratio $1 / \omega \tau$ and by a direct test in the classical regime using molecular dynamics by Bickham and Feldman (1998) and Bickham (1999)

It can be seen in Fig. 9 that no change occurs in the size of $1 / \tau$ at the mobility edge. A careful treatment of locons does not support the idea that they thermalize slowly. Their anharmonic thermalization rates (Fabian, 1997a) are as fast, or even faster, than diffusons, and also comparable to, or faster than, corresponding thermalization rates of vibrations in crystals. The source of the misconception of slow equilibration is the idea that direct decay of a locon into two locons should be negligible. This idea fails because, unlike for example, band-tail electronic states in amorphous $\mathrm{Si}$, the vibrational states are not at all dilute. Slow thermalization rates (forbidden by all theories we understand) could be tested by looking for a contribution from thermal vibrations to attenuation of very high-frequency sound (Fabian and Allen, 1999). If the thermalization rate is extremely slow, this contribution to the attenuation would be greatly enhanced.

\section{RESONANT MODES}

Inspection of Fig. 6 shows that a few modes in the D region and somewhat more in the $\mathrm{P}$ region have anomalously small participation ratios, of order 100 out of the 1000 atoms available. These states are not exponentially localized (Fabian, 1997b; Feldman et al., 1999) but are temporarily trapped in regions of peculiar coordination, from which they can tunnel into the continuum of extended states. Such states were first reported by Biswas et al. (1988) in a small model similar to ours; a model with larger numbers of 3 - and 5-fold coordinated atoms had more such modes, which were speculated to bear some relation to the "floppy modes" of Phillips and Thorpe. Such modes were studied in detail by Schober and coworkers $(1988,1991)$ and Oligschleger and Schober (1999).

We have recently argued (Feldman et al., 1999) that, in our (mostly 4-fold coordinated) models of amorphous $\mathrm{Si}$, such states tend to disappear as the size of the model gets bigger, presumably because each such mode is trapped only in a very specific peculiar region. As the number of atoms in the model increases, so does the number of peculiar regions, but if each resonant mode is trapped in only one region, the fraction of time spent outside that region increases because the volume outside that region has increased. On the other hand, such modes, especially the ones in the $\mathrm{P}$ region, may be more pronounced in real amorphous $\mathrm{Si}$ and other real glasses than they are in the models we study. This is because our models are spatially homogeneous on scales greater than $4 \AA$, while real glasses may have mesoscopic defects such as voids which would attract more such modes.

Fabian and Allen $(1997,1999)$ found that the resonant modes have giant $(\approx-40)$ Grüneisen parameters $\gamma_{i}$. These parameters measure the sensitivity of $\omega_{i}$ to macroscopic strain. In a glass (just as in a crystal where positions of atoms are not all fixed by crystallographic symmetry) strains cause not just a homogeneous shift of atomic coordinates, but also an additional local relaxation, which turns out to be particularly large in just those peculiar regions where the resonant modes are temporarily trapped. Anomalously large values of $\gamma_{i}$ play an important role in explaining the anomalously large and sample-dependent measured low- $T$ thermal expansion of glasses, and also should show up in enhanced contributions to the attenuation of high $\omega$ sound waves at higher $T$.

\section{CHARACTER OF DIFFUSONS}

The most important property which distinguishes diffusons is their intrinsic diffusivity $D_{i}$ with values of order $\omega_{D} a^{2} / 3$. If wave-packets were constructed in such a way as to be approximately monochromatic, and simultaneously localized at the center of a cell with a reasonably 
small radius (perhaps $6-8 \AA$ ), then we believe that no matter how well directed such a pulse was at $t=0$, the center of the pulse would hardly move, and the radius would evolve as $\left\langle r^{2}\right\rangle=6 D t$ for all times until reaching the cell boundary where it would interfere with its periodic image. Unfortunately, a $44 \AA$ cell is only marginally big enough, and computational difficulties have so far prevented us performing this experiment. Here we describe our efforts to find other ways to characterize diffusons.

\section{A. wavevector}

At the P/D boundary, wavevectors become ill-defined. Fig. 10 shows a test. The squared Fourier weight is defined as

$$
w_{i}(\vec{Q})=\left|\sum_{\ell} e^{i \vec{Q} \cdot \vec{R}(\ell)} \vec{\epsilon}_{i}(\vec{R}(\ell))\right|^{2}
$$

where $\vec{Q}$ is chosen as $(2 \pi / L)(h, k, l)$ with integer $h, k, l$ so that the periodic images interfere constructively. We define $w_{i}(Q)$ as $w_{i}(\vec{Q})$ averaged over spherical shells of wave vector of width $0.2 \times 2 \pi / L$. The value $Q=9.2 \times 2 \pi / L$ corresponds to neighboring atoms being completely out of phase. The $51 \mathrm{meV}$ diffusons show a peak Fourier content near $8 \times 2 \pi / L$, but the peak height is less than twice a "background" value found at larger $Q$ which dominates the behavior. There is no ballistic character to these modes.

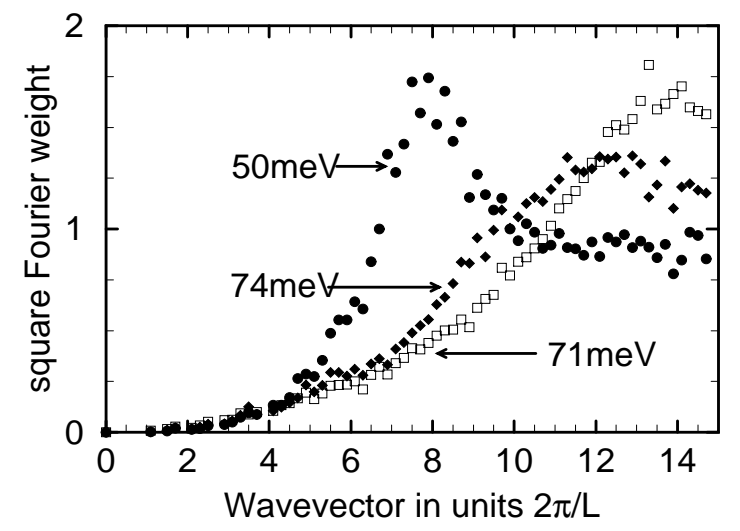

FIG. 10. Fourier weights calculated from Eq. 8 for diffusons at $50 \mathrm{meV}$ and $71 \mathrm{meV}$, and locons at $74 \mathrm{meV}$, averaged over 3,4 , and 2 modes respectively.

\section{B. polarization}

Diffusons have no wave vector. Not surprisingly, they also lack a polarization as is shown in Fig. 11. Propagons, by definition, have a wavevector. The nature of the propagons in the 4096 atom model was examined by Feldman et al. (1999). As shown there, the modes near $\omega=3.5 \mathrm{meV}$ have well-defined TA character, with the smallest possible wave vector $Q=2 \pi / L$. Fig 11 shows that these modes have only limited preference for a direction of polarization. Similarly, the mode at $7.2 \mathrm{meV}$ has $Q=2 \pi / L$ and LA character, but not much polarization.

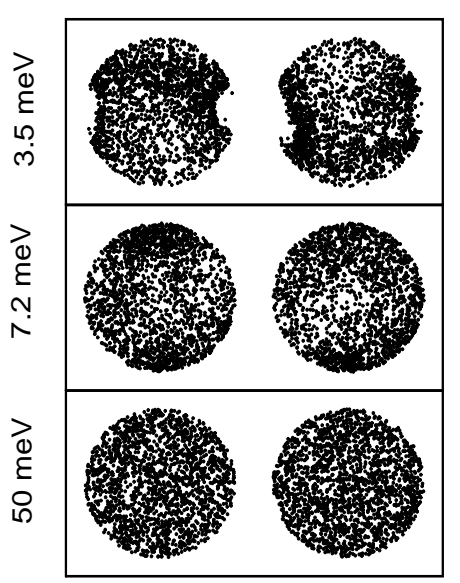

FIG. 11. Area-preserving projection normalized unit polarizations $\hat{\epsilon}_{i}(\vec{R}(\ell))$ onto circles (one circle for each hemisphere.) Three modes $i$ are shown, with each of the 4096 components showing as a dot.

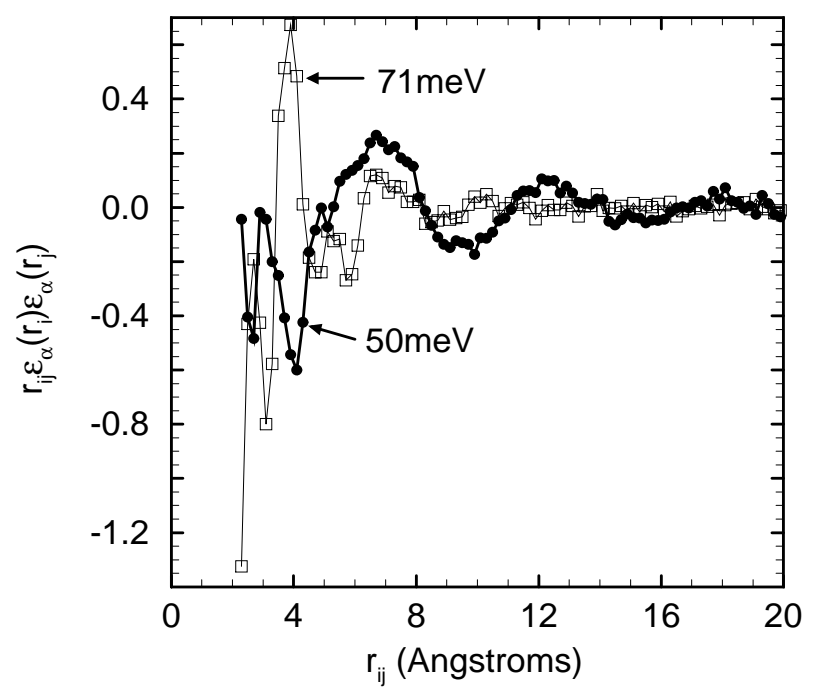

FIG. 12. The spatial fall-off of $\vec{\epsilon}\left(\vec{r}_{i}\right) \cdot \vec{\epsilon}\left(\vec{r}_{j}\right)$ weighted by $r_{i j}$ and averaged for the same 3 modes at $50 \mathrm{meV}$ and 4 modes at $71 \mathrm{meV}$ as in fig. 5 . 
Polarization directions of diffusons wander uniformly over the unit sphere, and one may ask what is the spatial range of decay of polarization memory in a mode. This is shown in Fig. 12. In a crystal, one has surfaces in $Q$-space where modes have constant frequency $\omega(\vec{Q})=$ const. A single eigenstate is some arbitrary linear combination of the degenerate Bloch waves on this surface. If the surfaces were spherical and the linear combinations random, we would expect that $\vec{\epsilon}\left(\vec{r}_{i}\right) \cdot \vec{\epsilon}\left(\vec{r}_{j}\right)$ would fall off spatially as $\cos \left(Q r_{i j}\right) / r_{i j}$. Fig. 12 shows that for diffusons at $50 \mathrm{meV}$, some polarization memory, but much less than expected for a crystal, persists out to $12 \AA$, while higher $\omega$ diffusons lose polarization memory more rapidly.

\section{C. phase quotient}

The "phase quotient" $\phi_{i}$ was defined by Bell and Hibbins-Butler (1975) as

$$
\phi_{i}=\frac{\sum_{<a, b>} \vec{\epsilon}_{i}(a) \cdot \vec{\epsilon}_{j}(b)}{\sum_{<a, b>}\left|\vec{\epsilon}_{i}(a) \cdot \vec{\epsilon}_{j}(b)\right|}
$$

and is plotted in Fig. 13. For low $\omega_{i}$, values near 1 indicate that nearest neighbor atoms (the only ones summed in Eqn. 9) vibrate mostly in-phase, while for high $\omega_{i}$, values near -1 indicate that nearest neighbors vibrate mostly out-of-phase. Like so many other properties, this depends only on $\omega_{i}$ until the $\mathrm{E} / \mathrm{L}$ boundary is crossed, but is very mode-specific for locons. The sharp rise at $\omega_{i} \approx 29 \mathrm{meV}$ is interesting, and may help explain why at the same frequency $D_{i}$ (Fig. 7) has a sudden rise. In crystalline $\mathrm{Si}$, at approximately the same point in the spectrum, the TA branch ends and the density of states has a local minimum. Thus $29 \mathrm{meV}$ marks a point in the spectrum where diffusons change character from bond-bending (somewhat TA-like) with relatively high frequency because of large phase difference from atom to atom, to bond-stretching (somewhat LA-like) with not such a large phase difference but an equally high frequency because the bond-stretching forces are bigger than the bond-bending forces. Apparently the latter kind of mode has greater diffusivity by a factor 2 or more. In a crystal we attribute this to a larger group velocity of the LA branch and a smaller density of states for decay by elastic scattering. Neither of these properties can be properly invoked for diffuson modes in a glass, but apparently similar physics is somehow at work.

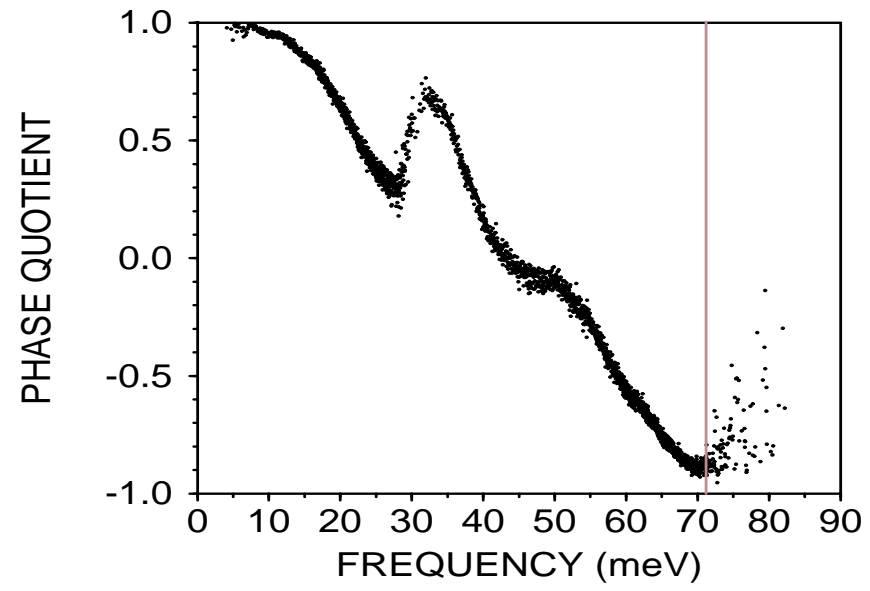

FIG. 13. Phase quotient versus energy for a 1000 atom model.

\section{SUMMARY}

Since $95 \%$ of the states in amorphous $\mathrm{Si}$, and probably many other glasses as well, are diffusons, we should understand their properties. All diffusons at a given frequency $\omega$ seem essentially identical. As $\omega$ changes, their properties evolve, mostly smoothly, but the sudden jump in diffusivity and in phase quotient at $29 \mathrm{meV}$ shows that not all variability is lost.

\section{ACKNOWLEDGMENTS}

We thank S. Bickham and K. Soderquist for relaxing the coordinates, and B. Davidson for providing eigenvalues and eigenvectors of the 4096 atom model. Work of PBA was supported in part by NSF grant no. DMR9725037. Work of JF was supported by the U. S. Office of Naval Research.

\section{REFERENCES}

Alexander, S., Laermans, C., Orbach, R., and Rosenberg, H. M., 1983, Phys. Rev. B 28, 4615; R. Orbach and A. Jagannathan, 1993, J. Phys. Chem. 98, 7411.

Alexander, S., 1989, Phys. Rev. B 40, 7953.

Allen, P. B., and Feldman, J. L., 1993, Phys. Rev. B 48, 12581.

Allen, P. B., and Kelner, J., 1998 Am. J. Phys. 66, 497.

Anderson, P. W., Halperin, B. I., and Varma, C. M., 1972, Philos. Mag. 25, 1.

Bell, R. J., and Dean, P., 1970, Disc. Faraday Soc. 50, 55. 
Bell, R. J., and Hibbins-Butler, D. C., 1975, J. Phys. C 8, 787.

Bickham, S. R., and Feldman, J. L., 1998, Phys. Rev. B 57, 12234 and Phil. Mag. B 77, 513.

Bickham, S. R., 1999, Phys. Rev. B 59, 4894.

Birch, F., and Clark, H., 1940, Am. J. Sci. 238, 529 and 612.

Biswas, R., Bouchard, A. M., Kamitakahara, W. A., Grest, G. S., and Soukoulis, C. M., 1988, Phys. Rev. Letters 60, 2280.

Bouchard, A. M., Biswas, R., Kamitakahara, W. A., Grest, G. S., and Soukoulis, C. M., 1988, Phys. Rev. B 38, 10499.

Cahill, D. G., Fischer, H. E., Klitsner, T., Swartz, E. T., and Pohl, R. O., 1989, J. Vac. Sci. Technol. A 7, 1259.

Cahill, D. G., Katiyar, M., and Abelson, J. R., 1994, Phys. Rev. B 50, 6077.

Canisius, J., and van Hemmen, J. L., 1985, J. Phys. C 18, 4873 .

Carles, R., Zwick, A., Moura, C. and Djafari-Rouhani, M., 1998, Phil. Mag. 77, 397.

Cobb, M., Drabold, D. A., and Cappelletti, R. L., 1996, Phys. Rev. B 54, 12162.

Dell'Anna, R., Ruocco, G., Sampoli, M., and Viliani, G., 1998, Phys. Rev. Letters 80, 1236.

Dove, M. T., Giddy, A. P., Heine, V., and Winkler, B., 1996, Am. Mineral. 81, 1057.

Edwards, J. T., and Thouless, D. J., 1972, J. Phys. C. 5, 807 .

Elliott, R. J., and Leath, P. L., 1975, in Dynamical Properties of Solids, (eds. G. K. Horton and A. A. Maradudin), North Holland, Amsterdam; vol. 2, p.385.

Fabian, J., and Allen, P. B., 1996, Phys. Rev. Letters 77, 3839 .

Fabian, J., 1997a, Phys. Rev. B 55, R3328.

Fabian, J., 1997b, Ph.D. dissertation, SUNY Stony Brook.

Fabian, J., and Allen, P. B., 1997, Phys. Rev. Letters $\mathbf{7 9}, 1885$.
Fabian, J., and Allen, P. B., 1999, Phys. Rev. Letters 82, 1478.

Feldman, J. L., Kluge, M. D., Allen, P. B., and Wooten, F., 1993, Phys. Rev. B 48, 12589.

Feldman, J. L., and Kluge, M. D., 1995, Phil. Mag. B 71,641 .

Feldman, J. L., Allen, P. B., and Bickham, S. R., 1999, Phys. Rev. B 59, 3551.

Feldman, J. L., Bickham, S. R., Engel, G. E., and Davidson, B. N., 1998, Philos. Mag. B 77, 507.

Gurevich, V. L., 1986, Transport in Phonon Systems, Elsevier Science Pub. Co., Amsterdam.

Jäckle, J., 1981, in Amorphous Solids: Low Temperature Properties, (ed. W. A. Phillips) Springer Verlag, Berlin; p.135.

Kamitakahara, W. A., Soukoulis, C. M., Shanks, H. R., Buchenau, U., and Grest, G. S., 1987, Phys. Rev. B 36, 6539.

Karpov, V. G., Klinger, M. I., and Ignat'ev, F. N., 1983, Zh. Eksp. Teor. Fiz. 84, 760 [Sov. Phys. JETP 57, 439.]

Keating, P. N., 1966, Phys. Rev. 145, 637.

Kittel, C., 1948, Phys. Rev. 75, 972.

Kubo, R., 1957, J. Phys. Soc. Jpn. 12, 570.

Kugler, S., Pusztai, L., Rosta, L., Chieux, P., and Bellisent, R., 1993, Phys. Rev. B 48, 7685.

Lee, Y. H., Biswas, R., Soukoulis, C. M., Wang, C. Z., Chan, C. T., and Ho, K. M., 1991, Phys. Rev. B 43, 6573 .

Liu, X., White, B. E. Jr., Pohl, R. O., Iwanizcko, E., Jones, K. M., Mahan, A. H., Nelson, B. N., Crandall, R. S., and Veprek, S., 1997, Phys. Rev. Letters 78, 4419.

Mott, N. F., and Davis, E. A., 1971, Electronic Processes in Non-Crystalline Materials, Clarendon Press, Oxford; p.24.

Nakhmanson, S. M., and Drabold, D. A., 1998, Phys. Rev. B 58, 15325.

Oligschleger, C., and Schober, H. R., 1999, Phys. Rev. B 59, 811 . 
Orbach, R., 1996, Physica B 220, 231.

Parshin, D. A., 1994, Phys. Solid State 36, 991.

Phillips, J. C., 1980, Phys. Stat. Solidi (b) 101, 473.

Phillips, W. A., 1972, J. Low Temp. Phys. 7, 351.

Phillips, W. A., 1987, Rep. Prog. Phys. 50, 1657.

Pohl, R. O., 1998, in Encylopedia of Applied Physics, Wiley-VCH, vol. 23, p.223.

Pompe, G. and Hegenbarth, E., 1988, Phys. Status Solidi B 147, 103.

Schirmacher, W., Diezemann, G., and Ganter, C., 1998, Phys. Rev. Letters 81, 136.

Schober, H. R., and Laird, B., 1991, Phys. Rev. B 44, 6746.

Schober, H. R., and Oligschleger, C., 1996, Phys. Rev. B 53, 11469 (1996).

Scholten, A. J., and Dijkhuis, J. I., 1996, Phys. Rev. B 53, 3837 .

Scholten, A. J., Akimov, A. V., and Dijkhuis, J. I., 1996, Phys. Rev. B 54, 12151.

Sette, F., Krisch, M., Masciovecchio, C., Ruocco, G., and Monaco, G., 1998, Science P280, 1550.

Sheng, P., and Zhou, M., 1991, Science 253, 539.

Sheng, P., Zhou, M., and Zhang, Z.-Q., 1994, Phys. Rev. Letters 72, 234.

Stillinger, F. H., and Weber, T. A., 1985, Phys. Rev. B $\mathbf{3 1}, 5262$.

Stolen, R. H., 1970, Phys. Chem. Glasses 11, 83.

Taraskin, S. N., and Elliott, S. R., 1997, Phys. Rev. B 56, 8605 .

Thorpe, M. F., 1983, J. Non-Cryst. Solids 57, 355.

Trachenko, K., Dove, M. T., Hammonds, K. D., Harris, M. J., and Heine, V., 1998, Phys. Rev. Letters 81, 3431.

Visscher, W. M., and Gubernatis, J. E., 1980, in Dynamical Properties of Solids, (eds. G. K. Horton and A. A. Maradudin), North Holland, Amsterdam; vol. 4, p.63.

Weaire, D., and Taylor, P. C., 1980, in Dynamical Properties of Solids, (eds. G. K. Horton and A. A.
Maradudin), North Holland, Amsterdam; vol. 4, p.1.

Wooten, F., Winer, K., and Weaire, D., 1985, Phys. Rev. Letters 54, 1392.

Zeller, R. C. and Pohl, R. O., 1971, Phys. Rev. B 4, 2029. 